\title{
A general method for the detection of large CAG repeat expansions by fluorescent PCR
}

Jon P Warner, Lilias H Barron, David Goudie, Kevin Kelly, David Dow, David R Fitzpatrick, David J H Brock

\begin{abstract}
The expansion of a tandemly repeated trinucleotide sequence, CAG, is the mutational mechanism for several human genetic diseases. We present a generally applicable PCR amplification method using a fluorescently labelled locus specific primer flanking the CAG repeat together with paired primers amplifying from multiple priming sites within the CAG repeat. Triplet repeat primed PCR (TP PCR) gives a characteristic ladder on the fluorescence trace enabling the rapid identification of large pathogenetic CAG repeats that cannot be amplified using flanking primers. We used our method to test a cohort of 183 people from myotonic dystrophy families including unaffected subjects and spouses. Eighty five clinically affected subjects with expanded alleles on Southern blot analysis were all correctly identified by TP PCR. This method is applicable for any human diseases involving CAG repeat expansions.

(f Med Genet 1996;33:1022-1026)
\end{abstract}

Key words: fluorescent PCR; CAG repeat; myotonic dystrophy; TP PCR.

Human Genetics Unit, Molecular Medicine Centre, Western General Hospital, Edinburgh EH4 2XU, UK

J P Warner

L H Barron

D R Fitzpatrick

D J H Brock

Department of Pathology,

Ninewells Hospital and

Medical School

Dundee DD1 9SY, UK

D Goudie

Medical Genetics

Laboratory,

University of

Aberdeen Medical

School,

Aberdeen AB9 2ZD,

UK

K Kelly

East Anglian Region

Genetics Service,

Addenbrooke's

Hospital, Hills Road,

Cambridge CB2 2QQ

UK

D Dow

Correspondence to: Dr Warner.

Received 10 June 1996 Revised version accepted for publication 17 July 1996 For people apparently homozygous for small common repeat size, the presence of a large unamplifiable allele can be excluded by performing a Southern blot and probing with a locus specific fragment flanking or containing the CAG repeat. We have developed a simple fluorescent PCR system that can rapidly identify but not size the largest alleles for any disorder with a CAG repeat expansion. This will reduce the number of Southern blot analyses required by allowing the identification of people homozygous for smaller alleles. Samples with large CAG expansions identified by TP PCR will, however, still require Southern blotting if an accurate estimation of size is needed.

\section{Materials and methods}

PCR DESIGN

Traditionally, PCR amplification of these unstable DNA sequences involves the use of a pair of locus specific primers (P1, P2) that flank the variable trinucleotide sequence. Our PCR assay uses a specific flanking fluorescently labelled primer (P1) in concert with a pair of primers (P3, P4) which have a common $5^{\prime}$ sequence (tail) (fig 1). The common $5^{\prime}$ sequence or tail primer (P3) was selected from an artificially produced random DNA sequence. The selection criteria for the sequence of the tail primer were (1) that it should contain little or no self complementarity, (2) no complementarity with either (GCA) $)_{5}$ or (TGC) ${ }_{5}$, and (3) no homology to known human sequences. The paired primer (P4) has the sequence $(\mathrm{GCA})_{5}$ or $(\mathrm{TGC})_{5}$ at its $3^{\prime}$ terminus depending on the strand of the CAG repeat to be amplified.

In the early amplification cycles the repeat specific $3^{\prime}$ terminus of $\mathrm{P} 4$ primes at multiple sites within CAG repeat alleles giving rise to a mixture of products. Specificity is dictated by the fluorescent locus specific primer (P1). A 10:1 molar ratio of $\mathrm{P} 3$ to $\mathrm{P} 4$ ensures that primer $\mathrm{P} 4$ is exhausted in the early amplification cycles. This reduces priming at $(\mathrm{CAG})_{\mathrm{n}}$ sites internal to the PCR products produced in earlier rounds, which would result in a gradual shortening of the average PCR product size (fig 1). The phenomenon of maintaining the allele size distribution by using a combination of tailed primer pairs was first described by Neil and Jeffreys $^{2}$ in the context of satellite DNA repeat amplification. The primer P3 preferentially binds to the end of products from previous amplification rounds owing to the stabilising effect of the $5^{\prime}$ tail sequence. A long extension time is used to allow complete extension of the larger sized products within the PCR product mixture.
POLYMERASE CHAIN REACTION

DNA used for PCR amplification was extracted from venous blood leucocytes using standard DNA extraction methods. Primer sequences are shown in table 1 . The sequences of HD1 and DRPLA 2 were selected from the $\mathrm{HD}^{3}$ and DRPLA ${ }^{5}$ gene sequences respectively. $\mathrm{P} 1$ 
A

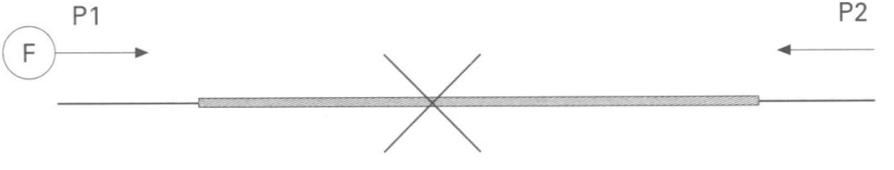

$\mathrm{B}$

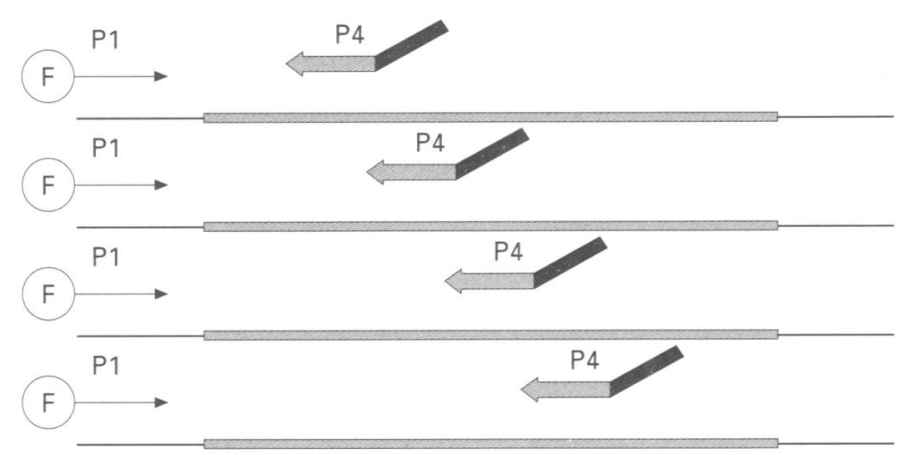

C

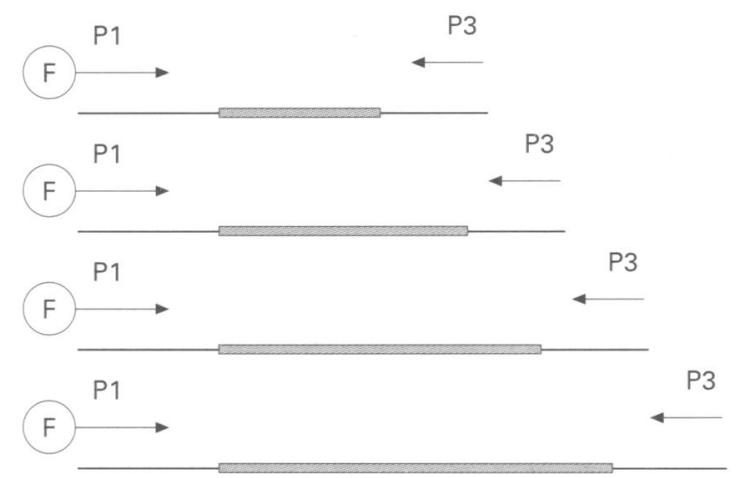

Figure 1 Design details for internally primed repeat specific PCR. Stippled box represents $(C A G)_{n}$ repeat. $F$ shows $5^{\prime}$ fluoresceinated primer. (A) For large alleles exceeding 100 $C A G$ repeats the $P C R$ using flanking primers $P 1$ and $P 2$ fails to give a product. (B) In the early amplification cycles primer the repeat specific $3^{\prime}$ terminus of $P 4$ binds at multiple sites within CAG repeat alleles giving rise to a mixture of products. Specificity is dictated by the fluorescent locus specific primer (P1). A 10:1 molar ratio of P3 to P4 ensures that primer P4 is exhausted in the early amplification cycles. (C) The primer P3 amplifies from the end of products from previous amplification rounds. A long extension time is used to allow complete extension of the larger sized products within the PCR product mixture and conserve the representation of the longer products.

and P2 were selected from the $3^{\prime}$ non-coding region of the myotonin protein kinase gene DNA sequence. ${ }^{1}$ The primers flank the myotonin CAG repeat, have matched annealing temperatures, and are also appropriate for amplification in conjunction with the primers $\mathrm{P} 3 \mathrm{R}$, P4CTG, and P4CAG. PCR reactions were performed in $25 \mu \mathrm{l}$ reaction volumes. The following buffer components were found to give optimal amplification: $1.5 \mathrm{mmol} / 1 \quad \mathrm{MgCl}_{2}$, $10 \mathrm{mmol} / 1 \mathrm{Tris} \mathrm{HCl}, \mathrm{pH} 8.3,50 \mathrm{mmol} / 1 \mathrm{KCl}$, $10 \% \mathrm{DMSO}, 200 \mu \mathrm{mol} / \mathrm{l} \mathrm{dATP}, 200 \mu \mathrm{mol} / 1$ dCTP, $200 \mu \mathrm{mol} / 1 \mathrm{dGTP}$, and $200 \mu \mathrm{mol} / 1$ dTTP. Genomic DNA (200 ng-1 $\mu \mathrm{g})$ and approximately $2 \mathrm{U}$ of $\mathrm{Taq}$ polymerase (Cetus) were used for each reaction. The following primer combinations were used. Mix A: P1 (5'

Table 1 Primer sequences

\begin{tabular}{ll}
\hline Primer & Sequence $\left(5^{\prime}-3^{\prime}\right)$ \\
\hline P1 & AGA AAG AAA TGG TTC TGT GAT CCC \\
P2 & GAA CGG GGC TCG AAG GGT CCT TGT AGC CG \\
P3R & TAC GCA TCC CAG TTT GAG ACG \\
P4CTG & TAC GCA TCC GAG TTT GAG ACG TGC TGC TGC TGC TGC T \\
P4CAG & TAC GCA TCC CAG TTT GAG ACG CAG CAG CAG CAG CAG CA \\
HD1 & ATG AAG GCC TTC GAG TCC CTC AAG TCC TTC \\
DRPLA 2 & TCA CCA TCA CCA CCA GCA ACA GCA A \\
\hline
\end{tabular}

fluoresceinated $1 \mu \mathrm{mol} / \mathrm{l})$ and P2 $(1 \mu \mathrm{mol} / \mathrm{l})$ for conventional amplification of the myotonic dystrophy CAG repeat, mix B: P1 ( $5^{\prime}$ fluoresceinated $1 \mu \mathrm{mol} / 1)$ with P3R $(1 \mu \mathrm{mol} / 1)$ and P4CTG $(0.1 \mu \mathrm{mol} / \mathrm{l})$, and mix C: P2 ( $5^{\prime}$ fluoresceinated $1 \mu \mathrm{mol} / \mathrm{l})$ with $\mathrm{P} 3(1 \mu \mathrm{mol} / \mathrm{l})$ and P4CAG $(0.1 \mu \mathrm{mol} / \mathrm{l})$ for amplifications priming from the CAG repeat allele. Samples were overlaid with mineral oil (Sigma) and incubated for four minutes at $94^{\circ} \mathrm{C}$ before the addition of the Taq polymerase. The reactions were subjected to 30 cycles of $94^{\circ} \mathrm{C}$ for one minute, $60^{\circ} \mathrm{C}$ for one minute, and $72^{\circ} \mathrm{C}$ for two minutes followed by a 10 minute extension at $72^{\circ} \mathrm{C}$ in a Perkin Elmer DNA thermal cycler.

Five microlitres of each PCR product were added to $5 \mu \mathrm{l}$ of distilled water and $10 \mu \mathrm{l}$ of loading buffer $(10 \mathrm{mg} / \mathrm{ml}$ blue dextran in deionised formamide) and incubated at $95^{\circ} \mathrm{C}$ for three minutes. Tubes were cooled on ice before loading on a denaturing $6 \%$ polyacrylamide $7 \mathrm{~mol} / \mathrm{l}$ urea gel. PCR products were resolved by electrophoresis in $0.6 \times \mathrm{TBE}$ and visualised using an automatic laser fluorescent sequencer (ALF Pharmacia). Traces were analysed using the Pharmacia fragment manager software.

SUBJECTS

Blood samples from 183 people from 48 myotonic dystrophy families had been collected by the Clinical Genetics Department in Edinburgh. DNA had been extracted and banked by the Molecular Genetics Service Laboratory in Edinburgh. DNA samples had been sent for Southern and conventional PCR analysis by another Scottish Molecular Genetics Consortium member laboratory in Aberdeen. This cohort included samples from unaffected people and spouses. The samples had been banked with no indication of the disease status of individual family members. The DNA samples were tested using PCR mixes A and $B$ and scored blind.

\section{Results and discussion}

Typical traces obtained using mix A are shown in fig 2. The PCR amplification across the myotonin CAG repeat gives reliable amplification up to alleles of approximately 100 CAG repeats but fails to amplify above this size. Analysis of severely affected myotonic dystrophy patients by genomic Southern blotting typically shows expansions between 1.5 and $4 \mathrm{~kb}$. PCR amplification of DNA from an affected subject carrying such an expansion gives a single normal allele and fails to pick up a larger allele, and is thus indistinguishable from patients genuinely homozygous for a small allele.

Typical traces obtained using mix $\mathrm{B}$ are shown in figs 3 and 4 . Mix B gives similar traces to mix C (not shown). Amplification products show a characteristic ladder with a three base pair periodicity when products are analysed on a laser fluorescence DNA sequencer. The ladder peaks at the size of the largest allele present or, for very large repeat alleles, the peak height diminishes gradually 


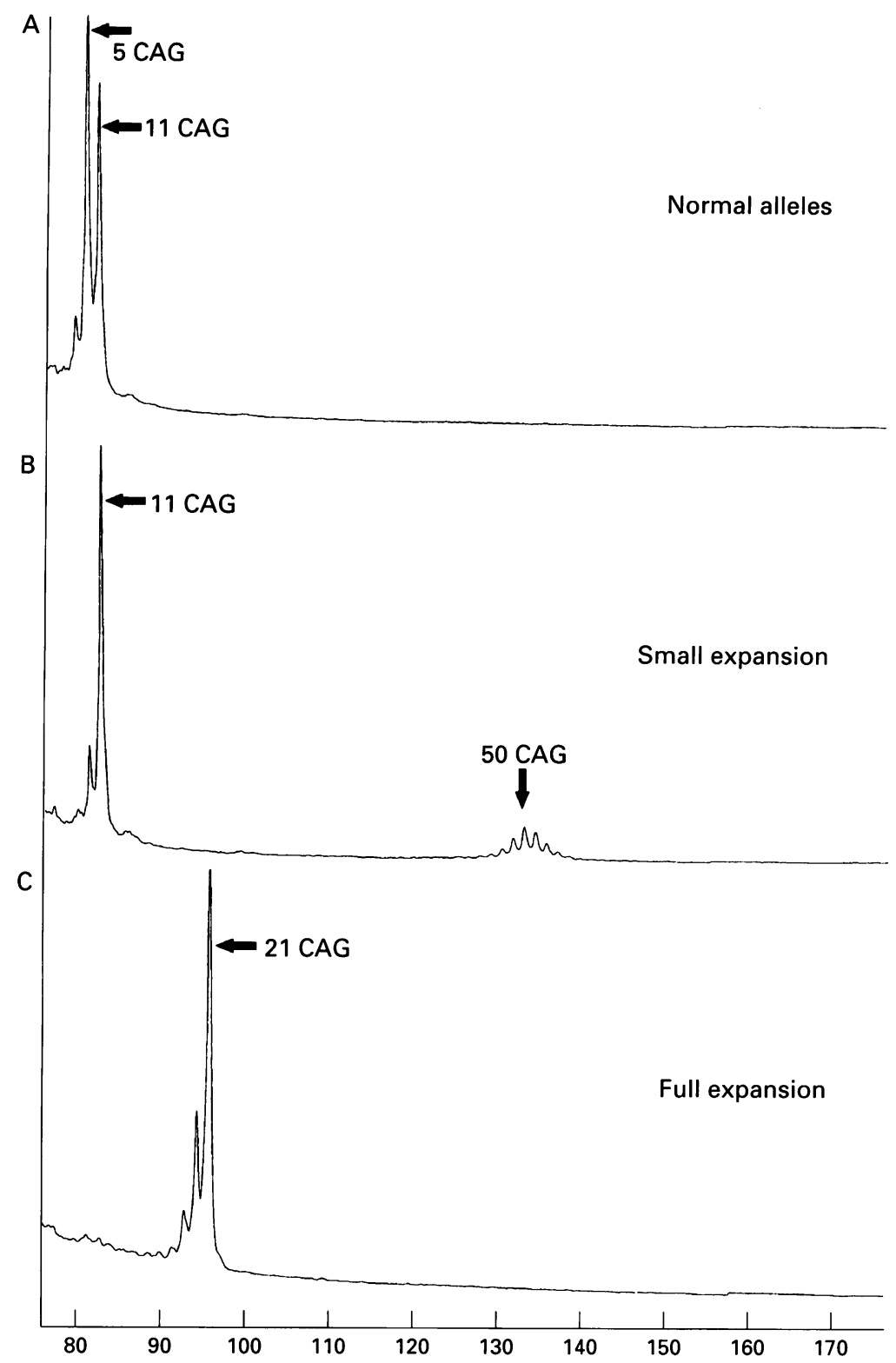

Figure 2 PCR amplification at the myotonin locus using flanking primers. The axis shows migration time in minutes. $C A G$ allele sizes shown with the arrows. (A) Trace obtained from a heterozygous normal subject. (B) Trace obtained from a heterozygous subject with a small expansion. (C) Trace obtained from a patient with myotonic dystrophy and an expanded allele size of $>4 \mathrm{~kb}$ as determined by Southern blot analysis. The larger allele fails to amplify. with mix A gave the characteristic ladder which extended beyond 50 CAG repeats with the peak height diminishing with increasing product size (figs 3 and 4, bottom trace). These 76 people were scored as affected with myotonic dystrophy with CAG repeat alleles of greater than 50 CAG repeats. The remaining 107 DNA samples did not give the long characteristic ladder seen for large CAG expansions with mix B. Amplification with mix A showed that 76 people were heterozygous for CAG repeat alleles in the normal range with 31 patients homozygous for alleles in the normal range.

The clinical status of the patients and the results of the PCR and genomic Southern analysis reported by the Aberdeen laboratory were consulted. A total of 74 of the 76 people with CAG repeat alleles in the affected range were symptomatic and classed with an affected disease status. Two patients had alleles just within the affected range and were as yet asymptomatic. Both had either children or close relatives affected with myotonic dystrophy in whom we had also detected expanded CAG repeat alleles. The results of the conventional DNA analysis were that 75 patients had expanded CAG alleles in the affected range, from $50 \mathrm{CAG}$ repeats to expansions of $>5 \mathrm{~kb}$. All 75 were in the group of 76 patients for whom our PCR assay had given evidence for the presence of a large CAG repeat allele. The one patient we identified who had not been ascertained by conventional testing was said to be clinically affected. The result of the Southern analysis for this DNA had been ambiguous because of poor DNA quality and a fresh sample will be collected for retesting.

On the basis of this series of tests we are confident that our TP PCR test for myotonic dystrophy is robust and reliable. For this large cohort of patients we obtained no false negative results and the results we obtained showed absolute concordance with the results obtained by conventional means. The only difference we saw suggested that our method was able to identify expanded alleles in poor quality DNA samples where Southern analysis failed to give a reliable result. This method does not measure the size of large expanded CAG repeat alleles but reliably identifies their presence. DNA samples can be screened using this method and only samples with large expansions would need to be analysed further using genomic Southern blotting in order to establish the exact size of large expanded alleles. The recently described XL PCR based method of Cheng et at could be used for samples where insufficient DNA for Southern analysis is available. This method is, however, technically demanding and labour intensive as PCR products are resolved by agarose gel electrophoresis, the gel being then blotted and probed with a locus specific probe. Our TP PCR method works if the fluorescent primer $\mathrm{P} 1$ is replaced by ${ }^{32} \mathrm{P}$ end labelled $\mathrm{P} 1$. These radiolabelled products are, however, more difficult to size accurately in the 30 to 60 CAG repeat size range (data not shown). Obviously silver staining is inappropriate for TP PCR as many non-specific inter(CAG) products are generated. 3 and 4 central trace) A further 72 samp that had given one normal CAG repeat allele 


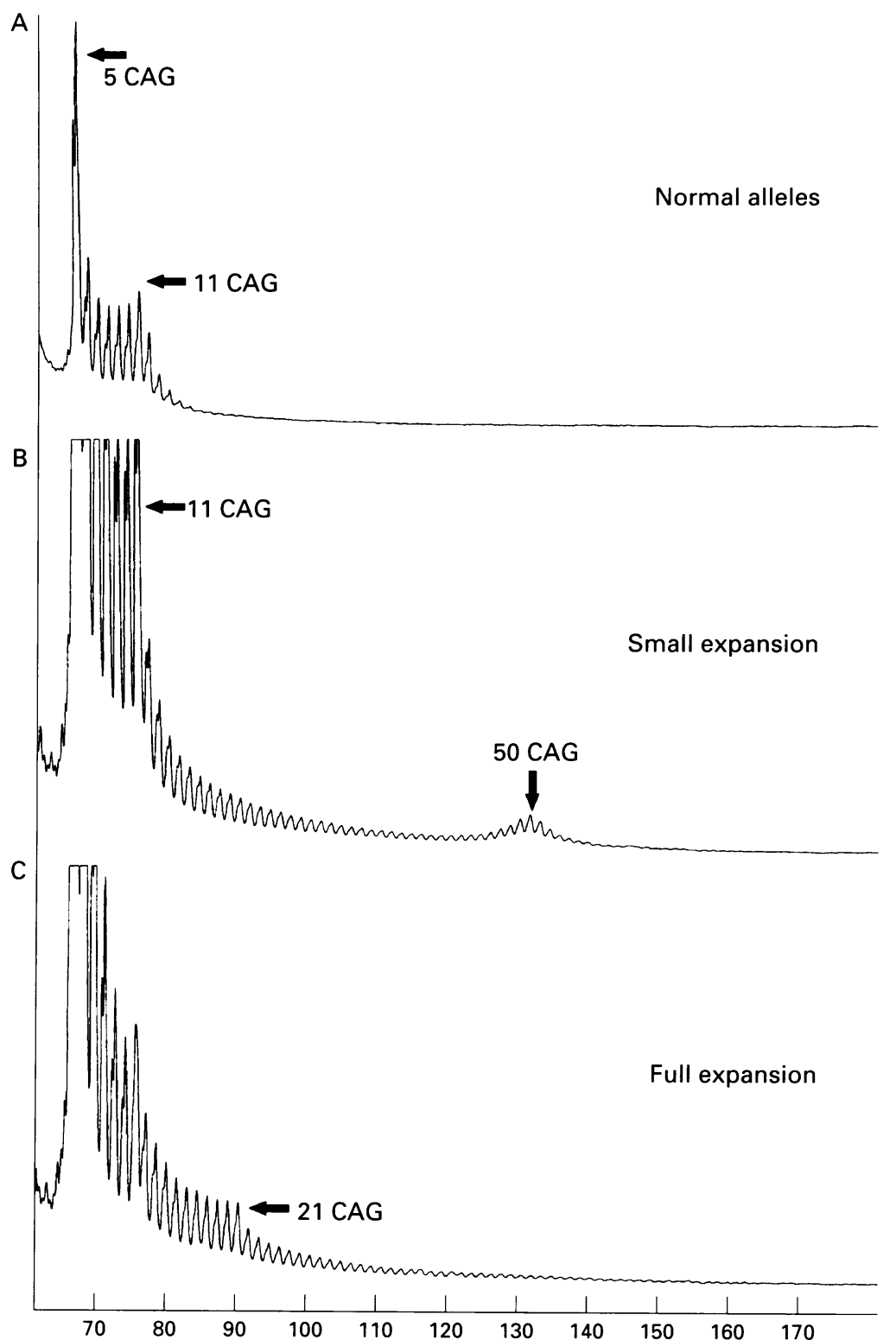

Figure 3.PCR amplification at the myotonin locus using internal primers. The axis shows migration time in minutes. CAG allele sizes shown with the arrows. The same people typed in fig 2 were retyped using mix $B$. Note the characteristic ladder with a $3 \mathrm{bp}$ periodicity. (A) Both alleles give peaks and all the intermediate priming sites give peaks. There is a slight continuation of the pattern beyond the maximum allele size owing to mispriming at the end of the repeat. This usually extends for less than $30 \mathrm{bp}$. (B) Both alleles give peaks as in $(A)$. (C) The ladder shows the presence of a large $C A G$ allele undetectable using flanking primers.
We have carried out some pilot TP PCR experiments using specific flanking primers for other diseases where expanded CAG repeats are the mutational mechanism. For Huntington's disease the same PCR conditions as described above for mix $B$ replacing the primer P1 with $\mathrm{HD}^{7}$ give reliable results (not shown). It has been suggested that for people with juvenile onset of Huntington's disease, where an expanded allele appears to be absent and the patient is homozygous for a smaller allele, a Southern analysis should be performed to exclude the presence of an unamplifiable large allele (Clinical Molecular Genetics Society of Great Britain, personal communication, 1995). Despite never having failed to amplify both alleles for juvenile Huntington's disease referrals up to 96 CAG repeats using flanking primers in Edinburgh, we would recommend the use of TP PCR to show the presence of these large, more difficult to amplify alleles. Similarly, we have shown that reliable results are obtained for large alleles for DRPLA when the primer DRPLA replaces P2 in mix C.

In conclusion we have developed a novel PCR assay that can be used for screening for expanded CAG repeat alleles including full mutation alleles of $>5 \mathrm{~kb}$ in myotonic dystrophy. TP PCR is robust and reliable and can in theory also be used in conjunction with locus specific flanking primers for any disease with a similar mutational mechanism.

We would like to thank all the clinicians who have sent us the samples over the years and Aileen Crosbie for help with clinical records.

(cont. overleaf) 

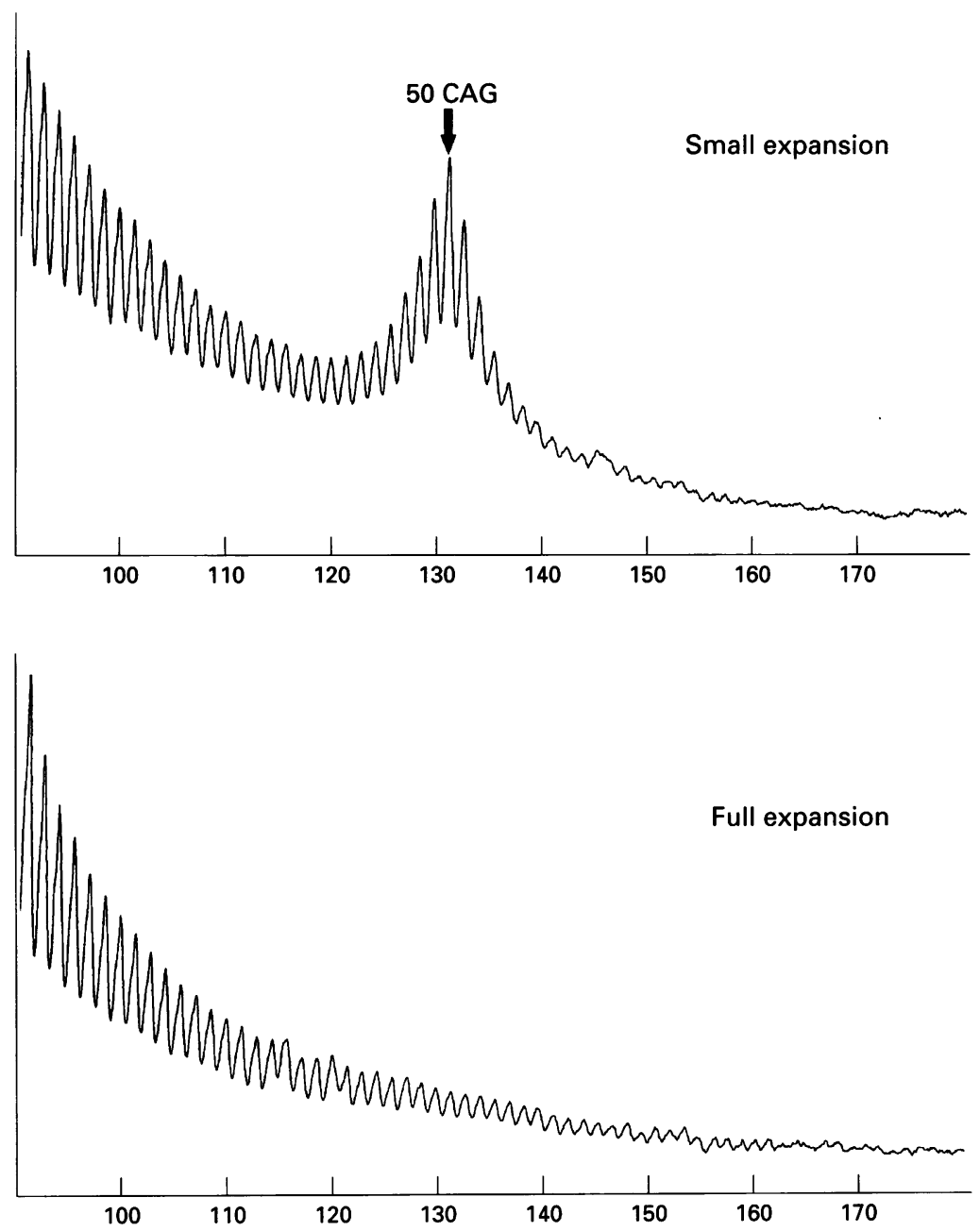

Figure 4 Close up of traces shown in fig 3. The axis shows migration time in minutes. $C A G$ allele sizes shown with the arrows. (A) Detail from trace shown in fig $3 B$. (B) Detail from trace shown in fig $3 C$.
1 Fu YH, Pizzuti A, Fenwick RG Jr, et al. An unstable triplet repeat in a gene related to myotonic muscular dystrophy. Science 1992;225:1256-8.

2 Neil DL, Jeffreys AJ. Digital DNA typing at a second hypervariable locus by minisatellite variant repeat mapping. Hum Mol Genet 1993;2:1129-35.

3 The Huntington's Disease Collaborative Research Group. A novel gene containing a trinucleotide repeat that is expanded and unstable on Huntington's disease chromosomes. Cell 1993;72:971-83.

4 Ashley CT, Warren ST. Trinucleotide repeat expansion and human disease. Annu Rev Genet 1995;29:703-28.

5 Nagafuchi S, Yanagisawa H, Sato K, et al. Dentatorubral and pallidoluysian atrophy: expansion of an unstable CAG trinucleotide on chromosome 12p. Nat Genet 1994;6:1418.

6 Cheng S, Barcelo JM, Korneluk RG. Characterisation of large CTG repeat expansions in myotonic dystrophy alleles large CTG repeat expansions in myotonic dystrophy alleles

7 Warner JP, Barron LH, Brock DJH. A new polymerase chain reaction (PCR) assay for the trinucleotide repeat that is unstable and expanded on Huntington's disease chromosomes. Mol Cell Probes 1993;7:235-9. 